\title{
Comparing Monte Carlo simulation and pseudospectral time-domain numerical solutions of Maxwell's equations of light scattering by a macroscopic random medium
}

\author{
Snow H. Tseng ${ }^{\text {a) }}$ and Boyeh Huang \\ Graduate Institute of Photonics and Optoelectronics and Department of Electrical Engineering, National \\ Taiwan University, No. 1, Sec. 4, Roosevett Road, Taipei 106, Taiwan
}

(Received 5 June 2007; accepted 10 July 2007; published online 1 August 2007)

\begin{abstract}
The Monte Carlo simulation of light scattering by a cluster of dielectric spheres is compared with numerical solutions of Maxwell's equations via the pseudospectral time-domain technique. By calculating the total scattering cross-section (TSCS) spectrum, respectively, the spectral light scattering characteristics are determined. Since the Monte Carlo simulation falls short to accurately account for coherent interference effects, it is shown that the Monte Carlo simulation yields TSCS spectra that significantly deviate from the numerical solutions of Maxwell's equations. Therefore, it is necessary to resort to Maxwell's equations in order to accurately determine the light scattering characteristics of a macroscopic geometry. (0) 2007 American Institute of Physics.
\end{abstract}

[DOI: $10.1063 / 1.2767777$ ]

The problem of light scattering through macroscopic random media is commonly found in nature, including nonperiodic structures such as clouds, biological tissues, etc. However, this problem has not been rigorously studied; due to the extreme complexity involved, heuristic approximations based on the radiative transfer theory ${ }^{1}$ are commonly employed. Among such heuristic methods, the Monte Carlo technique $^{2-6}$ is widely used for the problem of light scattering through macroscopic random media, particularly in the area of tissue optics.

However, all heuristic methods are fundamentally limited by the imposed hypotheses. For example, in the study of light scattering by random media, the Monte Carlo technique assumes independent scattering events between stochastic, pointlike scatterers; such assumptions involve modifications of the physical nature of the original problem, and fall short to accurately account for all the physics of the problem, including polarization and coherent interference. As a result, the validity and applicability of such heuristic methods remain to be determined. ${ }^{7,8}$ In order to accurately account for the coherence effects and near-field interactions, a rigorous research method based on Maxwell's equations is preferred.

In this letter, we simulate the light scattering characteristics of closely packed dielectric spheres by employing two different approaches: the Monte Carlo technique and the numerical solutions of Maxwell's equations via the pseudospectral time-domain (PSTD) technique, ${ }^{9}$ respectively. The Monte Carlo technique is a widely used heuristic approach based on the radiative transfer theory; by assuming light undergoes a sequence of independent scattering events, light scattering through random media is treated statistically. ${ }^{10,11}$ On the other hand, the PSTD technique is a numerical method where the light scattering problem is simulated by solving Maxwell's equations numerically. In this letter, we report the application of both simulation techniques, where the total scattering cross-section (TSCS) spectra are calculated and compared.

\footnotetext{
${ }^{a)}$ Electronic mail: snow@cc.ee.ntu.edu.tw
}

Light scattering by a cluster of dielectric spheres is simulated using the Monte Carlo technique. The Monte Carlo technique has been widely used in simulating light scattering through random media. ${ }^{2,6,12,13}$ For a specific wavelength, a total of 1000000 photons are injected into a cluster (cluster diameter $d=50 \mu \mathrm{m}$ ) of $N$ dielectric spheres; each sphere has a diameter $d=6 \mu \mathrm{m}$, with a refractive index $n$ $=1.2$. Each photon scattering angle is determined with a random number generator based on the Mie scattering phase function of a single dielectric sphere. The TSCS spectrum from 30 to $300 \mathrm{THz}$ is calculated.

Alternatively, we calculate the light scattering characteristics of a cluster of dielectric spheres by employing the PSTD technique. The PSTD simulation is a grid-based technique capable of solving Maxwell's equations numerically. By assigning the spatial distribution of the refractive index, the PSTD technique can accurately determine the light scattering characteristics of macroscopic geometries, including irregular geometries. In this letter, we report the employing of the PSTD technique on a parallel computer to model fullvector, three-dimensional (3D) scattering of light by a cluster of dielectric spheres in free space. A standard anisotropic perfectly matched layer absorbing boundary condition $^{14}$ is implemented to absorb outgoing waves to simulate light scattering in free space. A near-to-far-field transformation ${ }^{15}$ is employed, allowing scattered light for a broadband of wavelengths at all angles to be obtained in a single simulation. Based on Maxwell's equations, the PSTD simulation is essentially an idealized optical experiment in a noiseless environment, where the coherent effects can be accurately determined.

By employing the PSTD technique, light scattering by a cluster of closely packed dielectric spheres in free space is simulated, yielding the TSCS spectrum from 0.5 to $300 \mathrm{THz}$ $\left(\lambda_{0}=600 \mu \mathrm{m}-1 \mu \mathrm{m}\right)$ with a resolution of $0.5 \mathrm{THz}$. With a grid resolution of $0.33 \mu \mathrm{m}$, a PSTD simulation of light scattering by a $(60 \mu \mathrm{m})^{3}$ cluster typically takes $\sim 12 \mathrm{~h}$ with a parallel computer cluster of $202.4 \mathrm{GHz}$ Pentium 4 Xeon processors. 

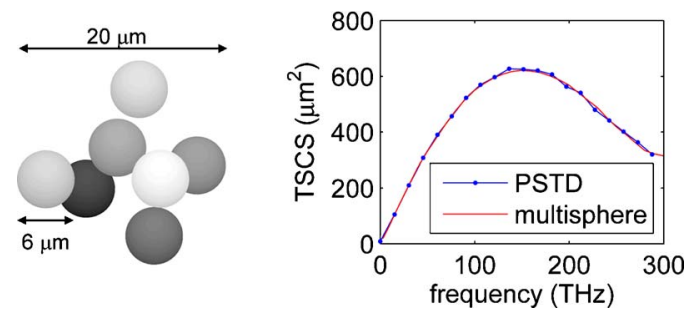

FIG. 1. (Color online) Validation of the 3D PSTD simulation. Light scattering by a cluster of seven randomly positioned, $6 \mu \mathrm{m}$ diameter, $n=1.2$ dielectric spheres is simulated, with a grid resolution of $d x=0.33 \mu \mathrm{m}$. The PSTD-computed total scattering cross-section (TSCS) as a function of frequency is compared with the multisphere expansion, which is based upon numerical expansions of Maxwell's equations.

A validation of the PSTD simulation of light scattering is shown in Fig. 1. Light scattering by seven randomly positioned, dielectric spheres is calculated using PSTD simulations and compared with the multisphere expansion. ${ }^{16}$ The TSCS spectrum is determined. As shown in Fig. 1, the PSTD-computed TSCS spectrum shows good agreement with the analytical expansions of Maxwell's equations.

The Monte Carlo technique and the PSTD technique are employed to simulate light scattering by a cluster of dielectric spheres, respectively. Firstly, as shown in Fig. 2, the TSCS spectra of a cluster of $N$ dielectric spheres are obtained using the Monte Carlo technique. Five cases are shown: $N$ $=25,50,75,125$, and 192. Notice that for sparse distribution of dielectric spheres (e.g., $N=25$ ), light impinging the cluster mostly encounters a single scattering event, and therefore the TSCS spectrum resembles the TSCS of a single dielectric sphere, showing that the Monte Carlo simulation yields results consistent with Maxwell's equations. However, as the cluster becomes closely packed with less space between adjacent spheres, the Monte Carlo assumption of "light undergoes independent scattering events" no longer holds and the

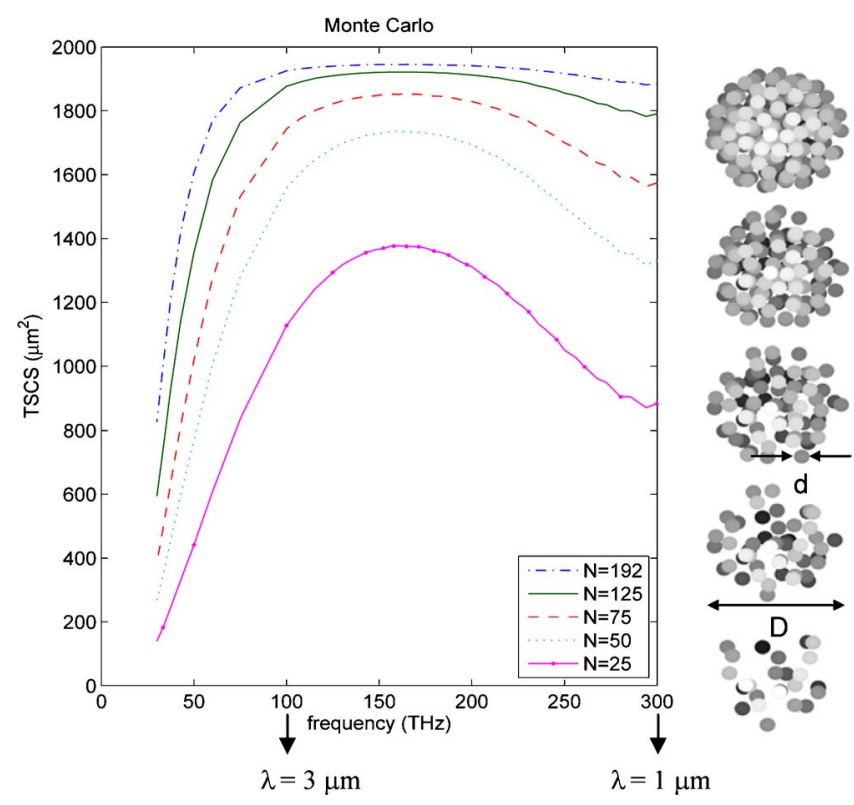

FIG. 2. (Color online) TSCS spectra obtained from the Monte Carlo simulation. By employing the Monte Carlo technique, the TSCS spectra are obtained, each corresponding to a (diameter $d=50 \mu \mathrm{m}$ ) cluster consisting of $N$ randomly positioned, $n=1.2$, (diameter $d=6 \mu \mathrm{m}$ ) dielectric spheres. Five

cases are shown (from bottom to top): $N=25,50,75,125$, and 192 . approximated as a stochastic sum of sequences of indepen-
Downloaded 13 Feb 2009 to 140.112 .113 .225 . Redistribution subject to AlP license or copyright; see http://apl.aip.org/apl/copyright.jsp
FIG. 3. (Color online) PSTD-computed TSCS spectra. Each TSCS spectra corresponds to a (overall diameter $d=50 \mu \mathrm{m}$ ) cluster consisting of $N$ randomly positioned, $n=1.2$, (diameter $d=6 \mu \mathrm{m}$ ) dielectric spheres. Five cases are shown (from bottom to top): $N=25,50,75,125$, and 192. Notice that as more dielectric spheres are packed together, the TSCS spectrum gradually exhibits optical characteristics due to the overall geometry which significantly differs from the TSCS spectrum obtained in the Monte Carlo simulation.

TSCS spectra obtained by the Monte Carlo simulation become less valid.

Secondly, as shown in Fig. 3, the TSCS spectra are calculated using the PSTD technique. By numerically solving Maxwell's equations, the TSCS spectrum of an arbitrary geometry is accurately determined. Notice that, due to the coherence effect, the magnitude of the TSCS spectrum based on Maxwell's equations is larger than the Monte Carlo results. Furthermore, as the cluster geometry becomes packed with more dielectric spheres, the TSCS spectra gradually exhibit optical characteristics that are significantly different from the Monte Carlo simulation (as shown in Fig. 2.)

To determine the relationship of the TSCS spectrum of a cluster of dielectric spheres and the size of the constituent spheres, we compare light scattering characteristics of two clusters, each consists of monodisperse dielectric spheres. As shown in Figs. 4(a) and 4(b), light scattering by a cluster of $d=6 \mu \mathrm{m}$ and $d=8 \mu \mathrm{m}$ dielectric spheres is simulated using the PSTD technique, respectively. Each $(60 \mu \mathrm{m})^{3}$ cluster consists of $N$ randomly positioned, closely packed, homogeneous, dielectric $(n=1.2)$ spheres of diameter $d$. Notice that even for an optically think cluster of closely packed dielectric spheres, the TSCS spectrum exhibits significantly different characteristics directly related to the size of the constituent spheres of each cluster.

Research findings show that the Monte Carlo simulation yields TSCS spectrum that significantly differs from the numerical solutions of Maxwell's equations, owing to the heuristic assumptions involved. Based on the radiative transfer theory, the Monte Carlo simulation of light scattering is treated as an energy transport problem by omitting the wave nature of light. Furthermore, light scattering through macroscopic random media (e.g., biological tissues) is heuristically approximated as a stochastic sum of sequences of indepenAIP license or copyright; see http://apl.aip.org/apl/copyright.jsp

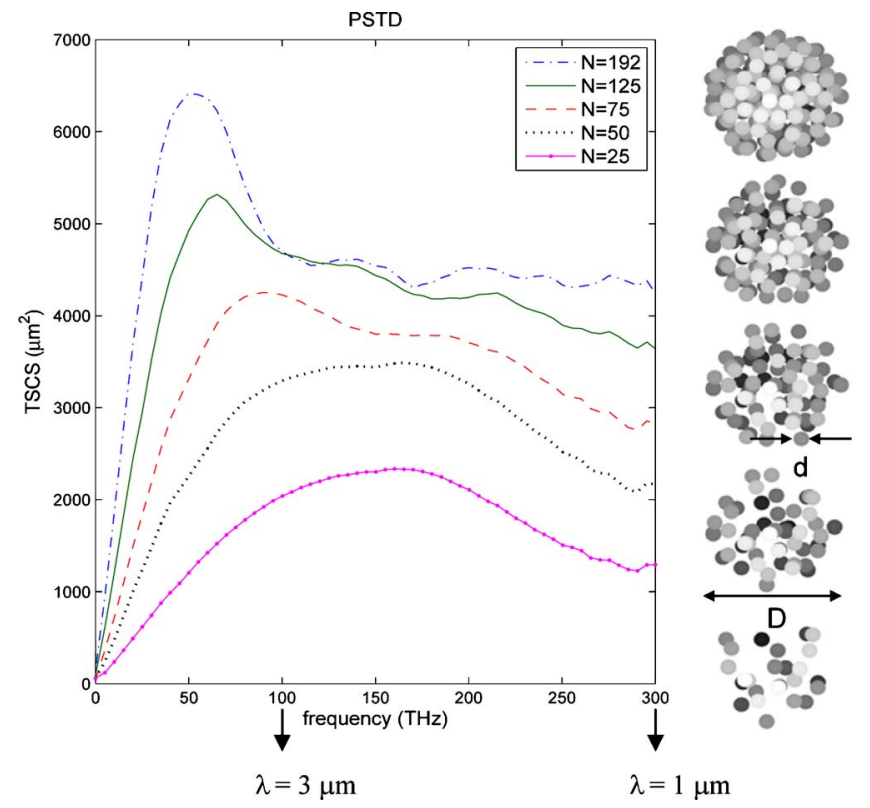




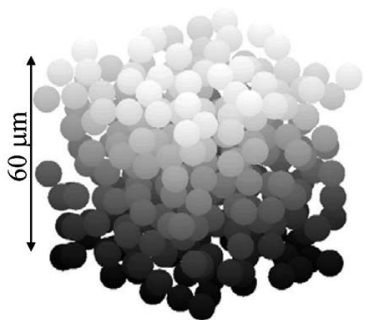

(a)
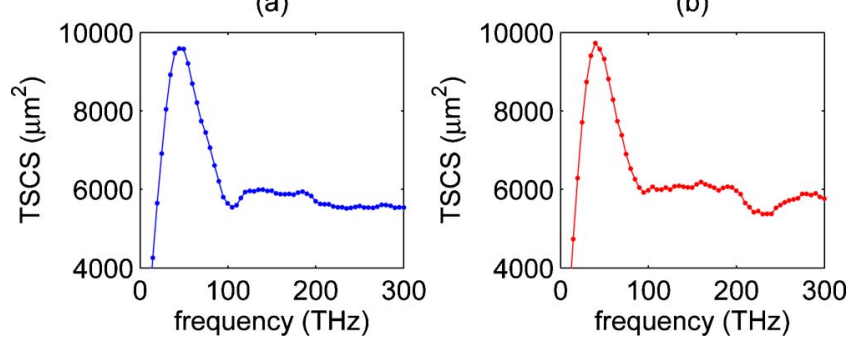

FIG. 4. (Color online) PSTD-computed TSCS spectra of a cubic cluster geometry. Each $(60 \mu \mathrm{m})^{3}$ cluster consists of $N$ randomly positioned, closely packed, dielectric $(n=1.2)$ spheres of diameter $d$. (a) $d=6 \mu \mathrm{m}, N=300$; (b) $d=8 \mu \mathrm{m}, N=140$.

dent scattering events. By omitting the wave nature of light, the complex light scattering problem is reduced to a simpler problem that is solvable but distorting the physics of the problem. As a result, such heuristic approximation in principle excludes the possibility of accurately determining the coherent effects of light using the Monte Carlo technique.

For sparse distribution of spheres (e.g., $N=25$ ), the Monte Carlo simulation yields a TSCS spectrum that is similar to the TSCS spectrum determined by the PSTD simulation based on Maxwell's equations, as shown in Fig. 2. This resemblance is anticipated, since with only a few dielectric spheres spaced far apart in space, the Monte Carlo assumption of independent scattering events is satisfied. However, for dielectric spheres closely packed in space, the assumption of light undergoes independent scattering events breaks down. By comparing Figs. 2 and 3, it is readily seen that the TSCS spectrum determined via the Monte Carlo simulation deviates significantly from the PSTD numerical solutions of Maxwell's equations. As the number of spheres increases and the cluster becomes closely packed, the overall TSCS spectrum is gradually dominated by the optical characteristics of the cluster as a whole and shows less of the characteristics due to individual spheres.

Specifically, (in Fig. 3) as the $N$ increases, a TSCS peak gradually forms around $55 \mathrm{THz}$; this peak is due to the coherent interference effects of the overall cluster geometry as a whole. ${ }^{17}$ For long wavelengths, the electromagnetic wave is insensitive to the microscopic structural details and reacts to the cluster geometry as a whole. This phenomenon is similar to the two-dimensional case, as reported in Ref. 17. In addition, notice that the amplitude of the Monte Carlo calculated TSCS spectra (Fig. 2) is significantly lesser than the PSTD calculated TSCS spectra (Fig. 3). This difference is anticipated because the Monte Carlo simulation treats each "photon" independently and does not accurately account for the coherent wave interference effects of light. As a result, the Monte Carlo simulation of light scattering falls short to determine the optical coherent effects.

Lastly, as shown in Fig. 4, the TSCS spectra exhibit optical characteristics that are related to the specific microscopic information of the cluster geometry (e.g., size of the constituent spheres). Such optical characteristics may provide essential information for innovative optical techniques. However, due to the complexity involved, a thorough analysis is required. Further analysis is currently ongoing and will be reported in future publications.

In summary, we report the comparison of the Monte Carlo simulation and PSTD numerical solutions of Maxwell's equations for the problem of light scattering by a cluster of monodisperse, dielectric spheres. Due to the heuristic assumptions, the Monte Carlo simulation falls short to account for the wave interference phenomenon. As a result, Monte Carlo simulation of light scattering yields optical characteristics that deviate from the numerical solutions of Maxwell's equations. On the other hand, based on Maxwell's equations, the PSTD technique is robust and applicable to light scattering problems of macroscopic arbitrary geometry. To accurately determine the coherent optical characteristics of macroscopic random medium, it is necessary to resort to Maxwell's equations.

The authors thank the Taiwan National Science Council under Grant No. 95-2112-M-002-039 for the support on this research. In addition, the authors would like to extend special thanks to all the computing facilities provided by the National Taiwan University Computing Center.

${ }^{1}$ A. J. Welch and M. J. C. van Gemert, Optical-thermal Response of Laserirradiated Tissue (Plenum, New York, 1995), p. 925.

${ }^{2}$ D. Boas, J. Culver, J. Stott, and A. Dunn, Opt. Express 10, 159 (2002).

${ }^{3}$ X. X. Guo, M. F. G. Wood, and A. Vitkin, Opt. Express 15, 1348 (2007).

${ }^{4}$ V. L. Kuzmin and I. V. Meglinski, Quantum Electron. 36, 990 (2006).

${ }^{5}$ I. Seo, J. S. You, C. K. Hayakawa, and V. Venugopalan, J. Biomed. Opt. 12 (2007).

${ }^{6}$ L.-H. Wang, S. L. Jacques, and L.-Q. Zheng, Comput. Methods Programs Biomed. 47, 131 (1995).

${ }^{7}$ L. Marti-Lopez, J. Bouza-Dominguez, J. C. Hebden, S. R. Arridge, and R. A. Martinez-Celorio, J. Opt. Soc. Am. A 20, 2046 (2003).

${ }^{8}$ M. Haney and R. Snieder, Phys. Rev. Lett. 91 (2003).

${ }^{9}$ L. H. Liu and P. F. Hsu, J. Quant. Spectrosc. Radiat. Transf. 105 (2007).

${ }^{10}$ X. Wang, G. Yao, and L.-H. Wang, Appl. Opt. 41, 792 (2002).

${ }^{11}$ J. C. Ramella-Roman, S. A. Prahl, and S. L. Jacques, Opt. Express 13, 4420 (2005).

${ }^{12}$ S. D. Campbell, A. K. O'Connell, S. Menon, Q. Su, and R. Grobe, Phys. Rev. E 74 (2006).

${ }^{13}$ Karri Muinonen, Waves Random Media 14, 365 (2004).

${ }^{14}$ S. D. Gedney, IEEE Trans. Antennas Propag. 44, 1630 (1996).

${ }^{15}$ A. Taflove and S. C. Hagness., Computational Electrodynamics: The finite-difference time-domain method (Artech House, Boston, 2000), p. 852.

${ }^{16}$ Y.-L. Xu and R. T. Wang, Phys. Rev. E 58, 3931 (1998).

${ }^{17}$ S. H. Tseng, J. H. Greene, A. Taflove, D. Maitland, V. Backman, and J. Walsh, Opt. Lett. 29, 1393 (2004). 